\title{
Analysis of recurrent event data with incomplete observation gaps using piecewise models ${ }^{\dagger}$
}

\author{
Yang-Jin $\mathrm{Kim}^{1}$ \\ ${ }^{1}$ Department of Statistics, Sookmyung Women's University \\ Received 22 May 2014, revised 5 July 2014, accepted 16 July 2014
}

\begin{abstract}
In a longitudinal study, subjects can experience same type of events repeatedly. Also, there may exist intermittent dropouts resulting in repeated observation gaps during which no recurrent events are observed. Furthermore, when such observation gaps have incomplete forms caused by the unknown termination times of observation gaps, ordinary approaches result in biased estimates. In this study, we investigate the effect of ignoring observation gaps and propose methods to overcome this problem. For estimating the distribution of unknown termination times, an interval-censored mechanism is applied and two cases are considered. Simulation studies are carried out to evaluate the performance of the proposed method. Conviction data of young drivers with several suspensions are analyzed to illustrate the suggested approach.
\end{abstract}

Keywords: Interval-censored data, observation gaps, piecewise-constant model, recurrent event data, YTOP.

\section{Introduction}

Recurrent event data can be found at diverse fields where a subject experiences same type of event repeatedly. Many authors have analyzed recurrent event data with several approaches (Andersen et al., 1993; Lawless and Nadeau, 1995; Lin et al., 2000). Cook and Lawless (2007) provided a comprehensive review for statistical analysis of recurrent event data. In this paper, our interest is to consider a special situation occurring at longitudinal study where a subject is assumed to be followed until a termination of study. However, some subjects can lose a follow-up and then return to the study after a while. Such intermittent dropouts are denoted as observation gaps or risk gaps (Zhao and Sun, 2006) and are regarded as non-risk status at survival analysis. Furthermore, when such observation gaps are observed with an incomplete form, the risk status becomes unclear. As a motivation of this paper, YTOP (Young Traffic Offence Program) data include 193 subjects' conviction record (Sun et al., 2001; Kim and Juhn, 2008; Kim, 2014). Among them, 40 ones experienced at least one suspension. The range of suspension per subject is $[0,7]$ and the suspended subjects were banned from driving cars during certain periods. Once a suspension began, subjects

$\dagger$ This work was supported by the Korea Research Foundation (MOEHRD, Basic Research Promotion Fund): (2012R1A1A3011350).

${ }^{1}$ Associate professor, Department of Statistics, Sookmyung Women's University, Seoul 140-742, Korea. E-mail: yjin@sookmyung.ac.kr 
dropped out of the risk state and came back to the risk state after completing the suspension. Therefore, the observation gap was determined by both start and termination of suspensions and the duration of observation gap depends on the termination time of suspension. With a complete information about observation gap, Therneau and Hamilton (1997) remarked on discontinuous intervals at risk in recurrent event data and utilized a counting process technique. However, at YTOP data, only starting time of suspension is available while the termination time is not known. Instead, the termination time was known to place somewhere between two time points. Therefore, to estimate the distribution of a unknown termination time, an interval-censored mechanism is considered. Kim and Jhun (2008) considered only one observation gap and derived an estimating equation. In this paper, we suggest a piecewise model for cases with several observation gaps under a total time scale. For a similar problem, Shin and Kim (2014) and Kim (2014) incorporate a gap time scale. Two cases are considered to estimate the incomplete termination time of observation gaps, (i) termination times are unrelated with covariates, and (ii) related with covariates. The reminder of this paper is organized as follows. In Section 2, we describe notations and models and Section 3 shows suggested methods and estimation procedures. Section 4 reports the results of simulation studies and in Section 5, the suggested method is applied to the YTOP dataset. The article concludes with some remarks in Section 6.

\section{Piecewise model with incomplete observation gaps}

Consider $n$ subjects experiencing recurrent events. Denote $T_{i j}$ as the $j$ th recurrent event time of subject $i\left(i=1,2, \ldots, n\right.$, and $\left.j=1, \ldots, n_{i}\right)$. Given a $p \times 1$ vector of time-dependent covariates, $x_{i}(t)$, the following intensity function is assumed,

$$
\lambda_{i}\left(t \mid x_{i}\right)=\lambda_{0}(t) \exp \left(\beta^{\prime} x_{i}(t)\right)
$$

where $\lambda_{0}(t)$ is an arbitrary baseline intensity function with a cumulative function $\Lambda_{0}(t)$ and $\beta$ is a vector of regression coefficients of $x_{i}(t)$. Define $Y_{i}(t)=I\left(t \leq C_{i}\right)$ as an atrisk indicator with a censoring variable, $C_{i}$. It is assumed that a censoring is independent of observation gaps as well as recurrent events. In order to incorporate observation gaps, denote $G_{i}=\left\{g_{i 1}, \ldots, g_{i m_{i}}\right\}$ as the set of observation gaps of subject $i$. Every $g_{i k}$ is composed of two time points under the $k$ th observation gap, $g_{i k}=\left[g s_{i k}, g t_{i k}\right]$, where $g s_{i k}$ and $g t_{i k}$ are a starting time and a termination time of the $k$ th observation gap of subject $i$, respectively. We consider a case where a termination time of observation gap is unknown while a starting time is available. Instead, the accessible information is that the termination occurred somewhere between two time points. In YTOP dataset, for example, $g t_{i k} \in\left(g s_{i k}, \tilde{t}_{i k}\right)$, where $\tilde{t}_{i k}$ is the event time occurring subsequently after the $k$ th suspension. If a study stops under the suspension, its termination time would be right censored, $g t_{i k} \in\left(g s_{i k}, \infty\right)$. Figure 2.1 shows an example of recurrent event data with two observation gaps. Termination time of the first observation gap is interval censored, $g t_{1} \in\left(g s_{1}, t_{2}\right)$ and one of the second observation gap is right censored, $g t_{2} \in\left(g s_{2}, \infty\right)$. For example, for $\mathrm{id}=139$ subject, there are 11 conviction recurrences and six suspensions, respectively, In detail, $t 1(=793)<t_{2}<t_{3}<t_{4}(=1934)<$ $g s_{1}(=2171)<t_{5}(=2181)<g s_{2}(=2210)<\cdots<t_{11}(=3036)<g s_{6}(=3057)<c_{i}(=3455)$. Then, $g t_{1} \in(2171,2181)$ and $g t_{6} \in(3057, \infty)$. 


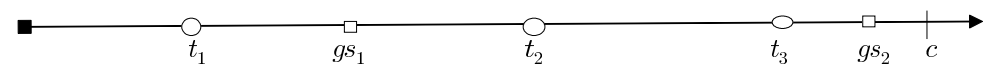

Figure 2.1 Observation gaps and recurrent event data scheme

In this study, a piecewise constant function for a baseline rate function is adopted. Lawless and Zhan (1998) and Liu and Huang (2009) showed that the pieces defined with appropriately selected cutpoints perform well at several situations. The application of piecewise constant model enables us to apply popular statistical packages such as R and SAS. Furthermore, the incorporation of piecewise function allows to check the influence of incompleteness through the following specification of cumulative intensities. Let $0=a_{0}<a_{1}<a_{2}<\cdots<a_{q}$ be $q$ cutpoints such that $a_{0}=0$ and $a_{q}=\tau=\max _{i} C_{i}$. Then the baseline intensity function is defined as follows,

$$
\rho(t ; \lambda)=\sum_{l=1}^{q} \lambda_{0 l} I\left(a_{l-1}<t \leq a_{l}\right)
$$

Without observation gaps, a cumulative intensity is defined by

$$
\Lambda_{0}\left(C_{i} ; \lambda\right)=\sum_{l=1}^{q} \lambda_{0 l} \max \left(0, \min \left(a_{l}-a_{l-1}, C_{i}-a_{l-1}\right)\right)=\sum_{l=1}^{q} \lambda_{0 l} u_{i l},
$$

where $u_{i l}$ is an exposure time by the $i$ th individual with a censoring time $C_{i}$ at the $l$ th interval $\left(a_{l-1}, a_{l}\right]$. With complete information for the observation gaps, the cumulative intensity is adjusted by the duration of observation gaps,

$$
\tilde{\Lambda}_{0}\left(C_{i} ; \lambda\right)=\sum_{l=1}^{q} \lambda_{0 l} \max \left(0, \min \left(a_{l}-a_{l-1}-d u r_{i l}, C_{i}-a_{l-1}-d u r_{i l}\right)\right)=\sum_{l=1}^{q} \lambda_{0 l} \tilde{u}_{i l}
$$

where $\tilde{u}_{i l}$ is the exposed time adjusted by observation gaps and $d u r_{i l}$ is calculated according to both the locations of observation gaps $\left(g s_{i k}, g t_{i k}\right)$ and the cutpoints $\left(a_{l} ; l=1, \cdots, q\right)$ as follows,

(i) $a_{l-1}<g s_{i k}<g t_{i k}<a_{l}$ :

$$
d u r_{i l}=g t_{i k}-g s_{i k},
$$

(ii) $a_{l-1}<g s_{i k}<a_{l}<g t_{i k}<a_{l+1}$ :

$$
d u r_{i, l}=a_{l}-g s_{i k}, d u r_{i, l+1}=g t_{i k}-a_{l},
$$

(iii $) a_{l-1}<g s_{i k}<a_{l}<a_{l+1}<g t_{i k}<a_{l+2}$ :

$$
d u r_{i l}=a_{l}-g s_{i k}, d u r_{i, l+1}=a_{l+1}-a_{l}, d u r_{i, l+2}=g t_{i k}-a_{l+1} .
$$

Define $e_{l}\left(t_{i j}\right)=I\left(a_{l-1}<t_{i j} \leq a_{l}\right)$ indicating that the $j$ th occurrence is observed in the $l$ th interval and $d_{l}=\sum_{i j} e_{l}\left(t_{i j}\right)$, a total number of the events occurring at the $l$ th interval. Let $\theta=\left(\beta, \lambda_{1}, \cdots, \lambda_{q}\right)$ be the vector of parameters of interest. Given complete data of recurrent 
events and observation gaps, a likelihood is written as follows,

$$
\begin{aligned}
L(\theta) & =\prod_{l=1}^{q} L_{l}(\theta) \\
& =\prod_{l=1}^{q} \lambda_{l}^{d_{l}} \prod_{i=1}^{n}\left\{\exp \left(\sum_{j=1}^{n_{i}}\left(x_{i}^{\prime}\left(t_{i j}\right) \beta\right) e_{l}\left(t_{i j}\right)-\lambda_{l} \tilde{u}_{i l} \int_{a_{l-1}}^{a_{l}} \exp \left(x_{i}^{\prime}(s) \beta\right) d s\right)\right\} .
\end{aligned}
$$

For estimating $\beta$, a profile likelihood is applied as follows (Cook and Lawless, 2007). A baseline intensity obtained through $\partial \log L(\theta) / \partial \beta$ is

$$
\tilde{\lambda}_{0 l}=\frac{d_{l}}{\sum_{r=1}^{n} \tilde{u}_{r l} \int_{a_{l-}}^{a_{l}} \exp \left(x_{r}^{\prime}(s) \beta\right) d s}, \quad l=1, \cdots, q
$$

and inserting $\tilde{\lambda}_{0 l}$ into $\log L(\theta)$ gives the following profile likelihood for $\beta$,

$$
L_{p}(\beta)=\prod_{i=1}^{n} \prod_{j=1}^{n_{i}}\left\{\frac{\exp \left(x_{i}^{\prime}\left(t_{i j}\right) \beta\right)}{\sum_{l=1}^{q} e_{l}\left(t_{i j}\right) \sum_{r=1}^{n} \tilde{u}_{r l} \int_{a_{l-1}}^{a_{l}} \exp \left(x_{r}^{\prime}(s) \beta\right) d s}\right\} .
$$

Then the solution to this equation is inserted into (2.3) and a baseline intensity is estimated $\hat{\lambda}$. However, equations (2.3) and (2.4) include incomplete data caused by unknown termination times. In next section, we consider the estimating procedure for estimating the distribution of unknown termination times.

\section{Interval censored termination times}

To recover incomplete data owing to unknown termination times, an interval censored mechanism is applied. It is assumed that the occurrence of observation gap is independent of recurrent event and the observation gaps occurring from a subject are independent of each other. Now, we introduce a new time scale to estimate the distribution of the termination times of observation gaps. Define $d_{i k}=g t_{i k}-\tilde{s}_{i k}$ as a renewal termination time, where $\tilde{s}_{i k}$ is an event time occurred just before the $k$ th observation gap. Remarks related with implementation of this time scale are discussed in Section 6. Since $g t_{i k}$ is either an intervalcensored or a right censored, $d_{i k}$ is also either an interval censored, $d_{i k} \in\left(t l_{i k}, t r_{i k}\right)$, where $t l_{i k}=g s_{i k}-\tilde{s}_{i k}$ and $t r_{i k}=\tilde{t}_{i k}-\tilde{s}_{i k}$ or a right censored, $d_{i k^{\prime}} \in\left(t l_{i k^{\prime}}, \infty\right)$. We assume that $d_{i k}$ is a discrete random variable with a probability mass function, $f_{i k r}=\operatorname{Pr}\left(d_{i k}=w_{r}\right)$ at suitably selected mass points, $\left\{w_{r}, r=1, \cdots, s\right\}$. Then $d_{i k}$ is estimated by

$$
E\left(d_{i k}\right)=\frac{\sum_{r=1}^{s} I\left(t l_{i k} \leq w_{r}<t r_{i k}\right) f_{i k r} w_{r}}{\sum_{r=1}^{s} I\left(t l_{i k} \leq w_{r}<t r_{i k}\right) f_{i k r}} .
$$

The above quantities are determined by $f_{i k r}^{\prime} s$ and we consider two approaches to estimates them; (a) termination occurs independently of covariates, (b) termination is related with covariates. 


\section{(a) Terminations are independent of covariates}

Under independence assumption, renewal termination times follow independent and identical distribution, $f_{i k l}=f_{l}$. To obtain suitable $w^{\prime} s$ and corresponding $f^{\prime} s$, a self-consistency algorithm (Turnbull, 1976) is applied. Let $w_{1}<\ldots<w_{s}$ be the admissible values of $d_{i k}, k=0,1, \cdots, m_{i}$, and $i=1, \cdots, n$ and can be constructed on the basis of the equivalence sets of $\left(t l_{i k}, t r_{i k}\right)$ (Turnbull, 1976; Lindsey and Ryan, 1998). Denote $\alpha_{i k l}$ as a binary indicator defining as, $\alpha_{i k l}=I\left(t l_{i k} \leq w_{l} \leq t r_{i k}\right)$. Then

$$
\hat{f}_{l}=\frac{\sum_{i=1}^{n} \sum_{k=1}^{m_{i}} v_{l}^{(i k)}}{\sum_{i=1}^{n} m_{i}}
$$

where

$$
v_{l}^{(i k)}=\frac{\alpha_{i k l} \hat{f}_{l}}{\sum_{m=1}^{q} \alpha_{i k m} \hat{f}_{m}} .
$$

Then with estimated $\hat{f}, E\left(d_{i k}\right)$ in (3.1) is calculated and corresponding termination times are estimated by $\hat{E}\left(g t_{i k}\right)=\hat{E}\left(d_{i k}\right)+\tilde{s}_{i k}$.

(b) Durations are related with covariates

For modeling the relation between termination times and covariates, a following proportional hazard model is applied,

$$
\gamma\left(d_{i k} ; \eta\right)=\gamma_{0}\left(d_{i k}\right) \exp \left(\eta^{\prime} z_{i k}\right)
$$

Several approaches can be considered to estimate regression coefficients for interval censored data (Pan, 2000; Finkelstein, 1986). In this study, a pseudo-likelihood approach suggested by Goetghebeur and Ryan (2000) is applied. Let $\tilde{Y}_{i k l}$ indicate whether $g_{i k}$ includes at risk at the $l$ interval and then $d N_{i k l}$ has a value one if $d_{i k}$ occurs at the $l$ th interval. Then a pseudo log likelihood is defined as follows,

$$
l_{p s}=\log L_{p s}=\sum_{i=1}^{n} \sum_{k=1}^{m_{i}} \sum_{l=1}^{s}\left\{\log \left(\gamma_{l}\right) d N_{i k l}+\eta^{\prime} z_{i k} d N_{i k l}-\gamma_{l} \exp \left(\eta^{\prime} z_{i k}\right) \tilde{Y}_{i k l}\right\} .
$$

By applying an EM algorithm, $\left(\left\{\gamma_{k}\right\}_{k=1}^{s}, \eta\right)$ are estimated and are applied to calculate a following probability mass function,

$$
\hat{f}_{i k r}=\hat{f}_{r}\left(z_{i k} ; \eta, \gamma\right)=\frac{p_{i k r}}{\sum_{t l_{i k} \leq w_{l} \leq t r_{i k}} p_{i k l}}, \quad t l_{i k} \leq w_{r} \leq t r_{i k}
$$

where $p_{i k l}=\hat{\gamma}_{l} \exp \left[\hat{\eta}^{\prime} z_{i k}-\exp \left(\hat{\eta}^{\prime} z_{i k}\right) \sum_{r=1}^{l} \hat{\gamma}_{r}\right]$. Using the estimated $\hat{f}_{i k r},(3.1)$ is estimated again by

$$
\hat{E}\left(\tilde{d}_{i k}\right)=\frac{\sum_{r=1}^{s} I\left(t l_{i k} \leq w_{r}<t r_{i k}\right) \hat{f}_{i k r} w_{r}}{\sum_{r=1}^{s} I\left(t l_{i k} \leq w_{r}<t r_{i k}\right) \hat{f}_{i k l}} .
$$

By inserting $\hat{E}\left(\tilde{d}_{i k}\right)$, the corresponding termination time is estimated by $\hat{E}\left(\tilde{g}_{i k}\right)=\hat{E}\left(\tilde{d}_{i k}\right)+$ $\tilde{s}_{i k}$. Now, the exposure times, $d u r_{i l}$ in $(2.2)$ are recalculated with $\hat{E}\left(\tilde{g} t_{i k}\right)$. Then, $\beta$ and $\lambda^{\prime} s$ are estimated using (2.3) and (2.4). For the variance of $\hat{\beta}$, the inverse of $I_{p}=-\partial^{2} l_{p}(\beta) /\left.\partial \beta \partial \beta^{\prime}\right|_{\beta=\hat{\beta}}$ is applied. 


\section{Simulation}

Simulation studies are carried out to evaluate the finite sample properties of the proposed method. To investigate the effect of proportion of observation gaps, several sampling schemes are applied. For each scheme, 300 datasets are generated with the following scheme; For subject $i$,

(i) Generate a binary covariate, $x_{i}=\{0,1\}$ with same probabilities.

(ii) For the $j(=1,2, \cdots)$ th recurrent event time, generate $u$ from a poisson process with an intensity function, $2.5 \exp \left(\beta x_{i}\right)$. Here, set $\beta=0.5$ Then, define a recurrent event time as $t_{i j}=t_{i j-1}+u+d u r$ with $t_{i 0}=0$, where dur is a duration time of observation gap.

(iii) For the $j$ th recurrent event, generate an indicator variable for an observation gap, $\delta_{j} \sim \operatorname{Ber}(p)$, where $p$ determines a proportion of the observation gaps occurring per a subject. In this study, we consider three values, $p=0.1,0.3$, and 0.5 . If $\delta_{j}=0$, then set $d u r=0$ and return to step (ii). Otherwise, the observation gap is generated as follows. Set $k=k+1$ and starting time and termination time are defined as $g s_{i k}=t_{i j}+u(0,0.01)$ and $g t_{i k}=g s_{i k}+d u r$, respectively. In this study, we consider two cases to evaluate the methods suggested in Section 3. (i) a termination time(or dur) is assumed to be independent of covariate. For distribution of dur, two distributions are applied, $d u r \sim U(0,1.0)$ or $\exp (1.0)$. (ii) a termination time(or dur $)$ is related with covariate. Therefore, a dur is generated from an exponential distribution with mean $0.5 \exp \left(\eta x_{i}\right)$, where $\eta=0.3$ or -0.3 . For $g t_{i k}>C_{i}, g t_{i k}$ is regarded as right-censored.

(iv) Iterate step (ii) and (iii) until $t_{i l}>C_{i}$, where a censoring time is generated from $C_{i}=6.00+U(0,1.5)$.

(v) For making an interval-censored termination time, set $L_{i k}=g t_{i k}-w_{1}, R_{i k}=g t_{i k}+$ $w_{2}, w_{1} \sim U(0,0.01)$ and $w_{2} \sim U(0,0.01)$. Then, define a renewal termination time as $g_{i k}=g t_{i k}-t_{i j}$ and corresponding interval times are $\left(t l_{i k}, t r_{i k}\right)=\left(L_{i k}-t_{i j}, R_{i k}-t_{i j}\right)$.

For all estimation procedures, the number of pieces is set as $q=5$. Table 4.1 and 4.2 show that simulation results of two methods: method ignoring observation gaps (Unadjusted) and the suggested method (Adjusted) when the termination of observation gaps are independent of covariate. The tables present estimates (est), mean of standard error (SEE), standard deviation of estimates (SSE), and the coverage probability of $95 \%$ confidence level (CP). According to results, the suggested method appears to have approximately unbiased estimates at all cases while unadjusted method gives biased estimates. In particular, as the proportion of observation gaps increases, biases are also getting bigger. Furthermore, when sample sizes increase, the biases tend to increase in unadjusted method. This can be explained that the increase of sample sizes results in more observation gaps which results in more biases. We found the sample deviation tends to be smaller than the standard error in unadjusted method even when the sample size $n$ increases.

Table 4.3 and 4.4 give the simulation results for cases where termination time is related with covariate. Estimated $\hat{\eta}$ seems to be unbiased for two values for $\eta=0.3$ or -0.3 . From Tables 4.3 and 4.4, the most noteworthy point is that the biases of $\hat{\beta}$ at $\eta=0.3$ are much smaller than those of $\eta=-0.3$ at the unadjusted method. It would be related with a 
positive value of $\eta$ which results in shorter duration times. Therefore, the influence of ignoring observation gaps at positive $\eta$ is much less than at negative $\eta$.

Table 4.1 Durations unrelated with covariate: Uniform distribution

\begin{tabular}{|c|c|c|c|c|c|c|c|c|c|}
\hline & & \multicolumn{8}{|c|}{$d u r \sim U(0,1.0)$} \\
\hline & & \multicolumn{4}{|c|}{ Unadjusted } & \multicolumn{4}{|c|}{ Adjusted } \\
\hline & $p$ & est & SEE & $\mathrm{SSE}$ & $\mathrm{CP}$ & est & SEE & SSE & $\mathrm{CP}$ \\
\hline \multirow{3}{*}{$n=50$} & 0.1 & 0.456 & 0.162 & 0.152 & 0.957 & 0.479 & 0.172 & 0.156 & 0.940 \\
\hline & 0.3 & 0.441 & 0.167 & 0.153 & 0.957 & 0.490 & 0.172 & 0.170 & 0.966 \\
\hline & 0.5 & 0.364 & 0.180 & 0.143 & 0.913 & 0.466 & 0.180 & 0.180 & 0.947 \\
\hline \multirow{3}{*}{$n=100$} & 0.1 & 0.463 & 0.113 & 0.106 & 0.950 & 0.494 & 0.113 & 0.110 & 0.960 \\
\hline & 0.3 & 0.417 & 0.121 & 0.103 & 0.923 & 0.496 & 0.121 & 0.116 & 0.960 \\
\hline & 0.5 & 0.369 & 0.126 & 0.099 & 0.857 & 0.479 & 0.126 & 0.118 & 0.967 \\
\hline \multirow{3}{*}{$n=200$} & 0.1 & 0.474 & 0.079 & 0.073 & 0.933 & 0.506 & 0.079 & 0.077 & 0.963 \\
\hline & 0.3 & 0.413 & 0.085 & 0.073 & 0.860 & 0.491 & 0.084 & 0.081 & 0.943 \\
\hline & 0.5 & 0.370 & 0.089 & 0.070 & 0.757 & 0.482 & 0.089 & 0.088 & 0.950 \\
\hline
\end{tabular}

Table 4.2 Durations unrelated with covariate: Exponential distribution

\begin{tabular}{cccccccccc}
\hline \hline \multicolumn{1}{c}{ Unadjusted } & \multicolumn{3}{c}{$d u r \sim \exp (1.0)$} \\
\hline & \multicolumn{1}{c}{ est } & SEE & SSE & CP & est & SEE & SSE & CP \\
\hline \multirow{3}{*}{$n=50$} & 0.1 & 0.476 & 0.112 & 0.112 & 0.936 & 0.493 & 0.112 & 0.113 & 0.950 \\
& 0.3 & 0.441 & 0.116 & 0.119 & 0.900 & 0.497 & 0.116 & 0.126 & 0.933 \\
& 0.5 & 0.402 & 0.121 & 0.108 & 0.800 & 0.483 & 0.121 & 0.119 & 0.947 \\
\hline \multirow{3}{*}{$n=100$} & 0.1 & 0.489 & 0.160 & 0.162 & 0.936 & 0.509 & 0.160 & 0.166 & 0.930 \\
& 0.3 & 0.441 & 0.167 & 0.153 & 0.946 & 0.499 & 0.166 & 0.164 & 0.960 \\
& 0.5 & 0.396 & 0.172 & 0.151 & 0.927 & 0.479 & 0.172 & 0.169 & 0.960 \\
\hline \multirow{3}{*}{$n=200$} & 0.1 & 0.474 & 0.079 & 0.075 & 0.963 & 0.495 & 0.079 & 0.078 & 0.953 \\
& 0.3 & 0.435 & 0.082 & 0.079 & 0.893 & 0.490 & 0.082 & 0.085 & 0.943 \\
& 0.5 & 0.416 & 0.084 & 0.078 & 0.863 & 0.494 & 0.085 & 0.086 & 0.933 \\
\hline
\end{tabular}

Table 4.3 Durations related with covariate: $n=50$

\begin{tabular}{|c|c|c|c|c|c|c|c|c|c|}
\hline & \multicolumn{4}{|c|}{ Unadjusted } & \multicolumn{5}{|c|}{ Adjusted } \\
\hline & \multicolumn{9}{|c|}{$d u r \sim \exp (0.3 z)$} \\
\hline$p$ & est & SEE & SSE & $\mathrm{CP}$ & est & SEE & SSE & $\mathrm{CP}$ & $\eta$ \\
\hline 0.1 & 0.474 & 0.163 & 0.143 & 0.970 & 0.499 & 0.159 & 0.163 & 0.950 & 0.352 \\
\hline 0.3 & 0.486 & 0.161 & 0.161 & 0.953 & 0.514 & 0.163 & 0.167 & 0.943 & 0.349 \\
\hline \multirow[t]{2}{*}{0.5} & 0.483 & 0.166 & 0.147 & 0.980 & 0.503 & 0.166 & 0.161 & 0.956 & 0.323 \\
\hline & \multicolumn{9}{|c|}{$d u r \sim \exp (-0.3 z)$} \\
\hline 0.1 & 0.470 & 0.160 & 0.154 & 0.966 & 0.503 & 0.160 & 0.158 & 0.966 & -0.399 \\
\hline 0.3 & 0.446 & 0.165 & 0.149 & 0.970 & 0.510 & 0.165 & 0.158 & 0.963 & -0.285 \\
\hline 0.5 & 0.396 & 0.168 & 0.162 & 0.896 & 0.496 & 0.169 & 0.177 & 0.933 & -0.306 \\
\hline
\end{tabular}

Table 4.4 Durations related with covariate: $n=100$

\begin{tabular}{|c|c|c|c|c|c|c|c|c|c|}
\hline & \multicolumn{4}{|c|}{ Unadjusted } & \multicolumn{5}{|c|}{ Adjusted } \\
\hline & \multicolumn{9}{|c|}{$d u r \sim \exp (0.3 z)$} \\
\hline$p$ & est & SEE & SSE & $\mathrm{CP}$ & est & SEE & SSE & $\mathrm{CP}$ & $\eta$ \\
\hline 0.1 & 0.478 & 0.115 & 0.108 & 0.953 & 0.506 & 0.111 & 0.108 & 0.963 & 0.305 \\
\hline 0.3 & 0.487 & 0.113 & 0.113 & 0.927 & 0.499 & 0.113 & 0.117 & 0.940 & 0.300 \\
\hline \multirow[t]{2}{*}{0.5} & 0.501 & 0.111 & 0.107 & 0.953 & 0.497 & 0.115 & 0.117 & 0.943 & 0.284 \\
\hline & \multicolumn{9}{|c|}{$d u r \sim \exp (-0.3 z)$} \\
\hline 0.1 & 0.478 & 0.111 & 0.117 & 0.920 & 0.498 & 0.112 & 0.119 & 0.933 & -0.319 \\
\hline 0.3 & 0.438 & 0.114 & 0.111 & 0.917 & 0.493 & 0.114 & 0.116 & 0.927 & -0.292 \\
\hline 0.5 & 0.407 & 0.117 & 0.102 & 0.900 & 0.494 & 0.117 & 0.110 & 0.953 & -0.283 \\
\hline
\end{tabular}




\section{Data analysis}

In this section, we applied the suggested method to the YTOP dataset (Sun et al., 2001). The original data set includes the conviction records of 193 young drivers who composed of 139 male drivers and 54 female ones. The range of convictions is $[0,13]$ and 40 drivers have at least one suspension and the range of suspension is $[0,7]$. However, the available information is only starting times of suspension but its termination times were unknown. In order to estimate the termination times of suspension, an interval censored mechanism is incorporated. Define a renewal termination time as $d_{i l}=g t_{i l}-\tilde{s}_{i l}$, where $\tilde{t}_{i l}$ is the recurrent event time occurred just before observation duration. Then, instead of knowing $d_{i l}$, we know $\left(t l_{i l}, t r_{i l}\right)$, where $t l_{i l}<d_{i l}<t r_{i l}, t l_{i l}=g s_{i l}-\tilde{s}_{i l}$ and $t r_{i l}=\tilde{t}_{i l}-\tilde{s}_{i l}$. The main interest of this dataset is to evaluate the effect of an educational program on reducing traffic convictions. Program participation was made in the middle of observation period and thus the participation to the program is a time dependent covariate. Define $x_{i 1}(t)=1, t \geq t_{0}$ where the $i$ th subject had experienced the educational program at $t_{0}$. With another covariate, gender $x_{i 2}=I(i$ th subject is male), interaction effect is considered. Table 5.1 presents the results of two methods; (i) Model 1: duration time is independent of covariates, and (ii) Model 2: duration time is related with covariates. In a comparison of two methods, the significance of YTOP on conviction recurrence is different even though they have same negative values. According to the result of Model 2, while YTOP participants and Male drivers have shorter duration times by positive values, their effects do not seem to be significant for reducing duration times of observation gap $\left(\hat{\eta}_{1}=0.288\right.$ and $\left.\hat{\eta}_{2}=0.148\right)$. Also compared with the likelihood values, Model 1 is preferred to Model 2. Therefore, the covarite effect on convictions is evaluated with Model 1 where a YTOP group has less convictions than Non-YTOP group and male drivers have more frequent convictions than female drivers. For five cutpoints, $a=(637,1096,1541,2018,3802)$, which are five quantiles, the estimates of baseline intensity are $\hat{\lambda}=(0.661,1.027,0.964,0.934,0.537) \times 10^{-3}$ for Model 1 . The number of cutpoints would be determined with the influence on the estimation of $\beta$. In our study, several other numbers of cutpoints still result in the similar estimates for $\beta$.

Table 5.1 YTOP data analysis with incomplete observation durations

\begin{tabular}{ccc}
\hline \hline & Model 1 & Model 2 \\
\hline YTOP (Program=1) & $-0.629(0.110)$ & $-0.164(0.113)$ \\
Gender (Male=1) & $1.432(0.102)$ & $1.5740(0.101)$ \\
$\eta[$ YTOP, Gender $]$ & & {$[0.288(0.377), 0.148(0.539)]$} \\
log-likelihood & 2978.16 & 2759.21 \\
\hline
\end{tabular}

\section{Discussion}

This paper considers a recurrent event data with repeated and incomplete observation gaps. For estimating unknown termination times of observation gaps, two assumptions about the relation between covariate and termination time are assigned. Instead, recurrent event of interest is independent with termination times and starting time of observation gaps. In real example, however, more recurrent events may cause more frequent occurrences of observation gap. Therefore, an association between two processes should be modelled and a multi-state process would be applied. Recently, the application of multi-state model to interval-censored data has been studied(Cook et al., 2008; Foucher et al., 2007). As another 
approach, for modelling an association between termination time and recurrent event data, a joint approach would be considered at future study (Liu et al., 2009). In this study, to estimate unknown termination times, renewal scale is applied to a termination time. As an alternative time scale, a calendar time could be applied to a termination time. Finally, another possible future study is to extend the suggested method to multivariate recurrent event data (Cook et al., 2010).

\section{References}

Andersen, P. K., Borgan, O., Gill, R. D. and Keiding, N. (1993). Statistical models based on counting processes, Springer, New York.

Cook, R., and Lawless, J. F. (2007). The statistical analysis of recurrent events, Springer, New York.

Cook, R., Lawless, J. F. and Lee, K. A. (2010) A copula-based mixed poisson model for bivariate recurrent events under event-dependent censoring. Statistics in Medicine, 29, 694-707.

Cook, R., Zeng, L. and Lee, K. (2008). A multistate model for bivariate interval-censored failure time data. Biometrics, 64, 1100-1109.

Finkelstein, D. (1986) A proportional hazards model for interval-censored failure time data. Biometrics, 42, 845-854.

Foucher, Y., Giral, M., Soulillou, J. P. and Daures, J. P. (2007) A semi-Markov model for multistate and interval-censored data with multiple terminal events. Application in renal transplantation. Statistics in Medicine, 26, 5381-5393.

Goetghebeur, E. and Ryan, L. (2000). Semiparametric regression analysis of interval-censored data. Biometrics, 56, 1139-1144.

Kim, Y. and Jhun, M. (2008). Analysis of recurrent event data with incomplete observation gaps. Statistics in Medicine, 27, 1075-1085.

Kim, Y. (2014). Regression analysis of recurrent events data with incomplete observation gaps. Journal of Applied Statistics. 41, 1619-1626.

Lawless, J. F. and Nadeau, J. C. (1995). Some simple robust methods for the analysis of recurrent events. Technometrics, 37, 158-168.

Lawless, J. F. and Zhan, M. (1999). Analysis of interval-grouped recurrent event data using piecewise constant rate functions. Canadian Journal of Statistics, 26, 549-565.

Lin, D. Y., Wei, L. J., Yang, I. and Ying, Z. (2000). Semiparametric regression for the mean and rate functions of recurrent events. Journal of Royal Statistical Society B, 62, 711-730.

Lindsey, J. and Ryan, L. (1998). Methods for interval censored data. Tutorial in biostatistics. Statistics in Medicine, 17, 219-138.

Liu, L. and Huang, X. (2009). Joint analysis of correlated repeated measures and recurrent events processes in the presence of a dependent terminal event. Applied Statistics, 58, 65-81.

Pan, W. (2000). Multiple imputation approach to Cox regression with interval censored data. Biometrics, 56, 199-203.

Shin, S. and Kim, Y. (2014). Statistical analysis of recurrent events with incomplete observation gaps. Journal of the Korean Data \& Information Science Society, 25, 327-336.

Sun, J., Kim, Y., Hewett, J., Johnson,.J. C., Farmer, J. and Gibler, M. (2001). Evaluation of traffic injury prevention programs using counting process approaches. Journal of the American Statistical Association, 96, 469-475.

Therneau, T. M. and Hamilton, S. C. (1997). rhDNase as an example of recurrent event analysis. Statistics in Medicine, 16, 2029-2047.

Turnbull, B. W. (1976). The empirical distribution function with arbitrarily grouped censored and truncated data. Journal of the Royal Statistical Society Series B, 38, 290-295.

Zhao, Q. and Sun, J. (2006). Semiparametric and nonparametric estimation of recurrent event with observation gaps. Computational statistics and data analysis, 51, 1924-1933. 